\title{
Prognostic and predictive value of $P D-L 2$ DNA methylation and mRNA expression in melanoma
}

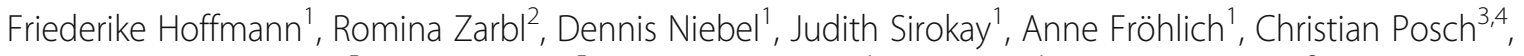
Tobias A. W. Holderried ${ }^{5}$, Peter Brossart ${ }^{5}$, Gonzalo Saavedra ${ }^{1}$, Pia Kuster ${ }^{1}$, Sebastian Strieth², Gerrit H. Gielen ${ }^{6}$, Sandra S. Ring ${ }^{7,8}$, Jörn Dietrich ${ }^{2}$, Torsten Pietsch ${ }^{6}$, Lukas Flatz ${ }^{8,9,10,11}$, Glen Kristiansen ${ }^{12}$, Jennifer Landsberg ${ }^{1 \dagger}$ and Dimo Dietrich ${ }^{2^{*}+}$ (1)

\begin{abstract}
Background: PD-L1 (programmed cell death 1 ligand 1) expression in melanoma has been associated with a better response to anti-PD-1 (programmed cell death 1) therapy. However, patients with PD-L1-negative melanomas can respond to anti-PD-1 blockade, suggesting that the other PD-1 ligand, PD-L2 (programmed cell death 1 ligand 2), might also be relevant for efficacy of PD-1 inhibition. We investigated PD-L2 expression and methylation as a prognostic and predictive biomarker in melanoma.

Methods: DNA methylation at five CpG loci and gene expression of PD-L2 were evaluated with regard to survival in 470 melanomas from The Cancer Genome Atlas. PD-L2 promoter methylation in correlation with PD-L2 mRNA and protein expression was analyzed in human melanoma cell lines. Prognostic and predictive value of PD-L2 methylation was validated using quantitative methylation-specific PCR in a multicenter cohort of 129 melanoma patients receiving anti-PD-1 therapy. mRNA sequencing data of 121 melanoma patients receiving anti-PD-1 therapy provided by Liu et al. were analyzed for PD-L2 MRNA expression.

Results: We found significant correlations between PD-L2 methylation and mRNA expression levels in melanoma tissues and cell lines. Interferon- $\gamma$ inducible PD-L2 protein expression correlated with PD-L2 promoter methylation in melanoma cells. PD-L2 DNA promoter hypomethylation and high mRNA expression were found to be strong predictors of prolonged overall survival. In pre-treatment melanoma samples from patients receiving anti-PD-1 therapy, low PD-L2 DNA methylation and high PD-L2 mRNA expression predicted longer progression-free survival.
\end{abstract}

Conclusion: PD-L2 expression seems to be regulated via DNA promoter methylation. PD-L2 DNA methylation and mRNA expression may predict progression-free survival in melanoma patients receiving anti-PD-1 immunotherapy. Assessment of PD-L2 should be included in further clinical trials with anti-PD-1 antibodies.

Keywords: PD-L1, PD-L2, DNA methylation, Melanoma, Prognostic biomarker, Predictive biomarker, Anti-PD-1 immunotherapy

\footnotetext{
* Correspondence: dimo.dietrich@gmail.com

${ }^{\dagger}$ Jennifer Landsberg and Dimo Dietrich contributed equally to this work.

${ }^{2}$ Department of Otolaryngology, Head and Neck Surgery, University Hospital Bonn, Venusberg-Campus 1, 53127 Bonn, Germany

Full list of author information is available at the end of the article
}

C C The Author(s). 2020 Open Access This article is licensed under a Creative Commons Attribution 4.0 International License, which permits use, sharing, adaptation, distribution and reproduction in any medium or format, as long as you give appropriate credit to the original author(s) and the source, provide a link to the Creative Commons licence, and indicate if changes were made. The images or other third party material in this article are included in the article's Creative Commons licence, unless indicated otherwise in a credit line to the material. If material is not included in the article's Creative Commons licence and your intended use is not permitted by statutory regulation or exceeds the permitted use, you will need to obtain permission directly from the copyright holder. To view a copy of this licence, visit http://creativecommons.org/licenses/by/4.0/. The Creative Commons Public Domain Dedication waiver (http://creativecommons.org/publicdomain/zero/1.0/) applies to the data made available in this article, unless otherwise stated in a credit line to the data. 


\section{Background}

Immune checkpoint inhibitors of the programmed cell death (PD-1) pathway are able to induce dramatic and durable regression of metastatic melanoma, consequently leading to their regulatory approval in metastatic melanoma and also recently in the adjuvant setting [1, 2]. However, despite the tremendous success of immune checkpoint blockade, the majority of patients do not benefit with long-term remissions, and a relevant proportion of patients suffers from long-lasting immunerelated side effects. Prognostic and predictive biomarkers are needed to identify patients at high risk of recurrence or progression and who are most likely to benefit from immunotherapy. To allow for the development of accurate predictive and prognostic biomarkers, knowledge on the regulation of immune checkpoint genes is mandatory.

PD-1 is a transmembrane receptor negatively regulating immune cells upon interaction with its two ligands PD-L1 (programmed cell death 1 ligand 1) or PD-L2 (programmed cell death 1 ligand 2). Receptor and ligands are key regulatory immune checkpoints that maintain selftolerance by adjusting the degree of activation of immune cells [3]. Although the basal expression of PD-L2 seems to be lower compared to PD-L1, its affinity to the PD-1 receptor has been shown to be 2-6-fold higher than that of PD-L1 in human T cells [4]. PD-L2 expression can be induced by inflammatory cytokines on different immune and non-immune cells $[5,6]$. Additionally, it can also be expressed by tumor cells including melanoma $[7,8]$. PD-L1 protein expression measured by immunohistochemistry (IHC) has shown a positive correlation with response to anti-PD-1 blockade in multiple studies in a variety of tumor entities $[9,10]$. However, patients with PD-L1 negative tumors can also benefit from anti-PD-1 blockade. Intratumoral heterogeneity of PD-L1 expression, the dynamic nature of PD-L1 expression in the tumor microenvironment, and the variability of detection methods can be explanations of the insufficiency of PD-L1 as a biomarker. Additionally, it has recently been shown that glycosylation of PD-L1 hinders its accurate immunohistochemical detection [11]. Other potential biomarkers, like density, phenotype, and diversity of tumor-infiltrating lymphocytes (TILs), tumor mutational burden, rare JAK2 or B2M mutations, and specific gut microbial species can also correlate with response to anti-PD-1 therapy, but remain imperfect predictors of a response to PD-1 blockade [12]. The role for PD-L2 in predicting response to anti-PD-1 therapy has barely been investigated $[9,13]$. Recently, in a cohort of pembrolizumab-treated patients with head and neck squamous cell carcinoma, PD-L2 positivity was significantly associated with response independent of PD-L1 status, and overall response rate was greatest in patients expressing both PD-L1 and PD-L2 ligands [8]. In patients with metastatic melanoma, PD-L1 and PD-L2 expression detected by IHC was associated with improved overall survival [7]. So far, the epigenetic regulation with particular focus on DNA promoter methylation of the PD-L2 encoding gene, PDCD1LG2, has not been considered as a biomarker in the context of anti-PD-1 immunotherapies in melanoma.

DNA methylation is an important epigenetic mechanism regulating the expression of proteins fundamental for $\mathrm{T}$ cell differentiation and $\mathrm{T}$ cell exhaustion [14-16]. Additionally, aberrant DNA methylation is an epigenetic hallmark of cancer and contributes to tumor progression by inactivating tumor suppressor genes [17]. It can function as a powerful biomarker that can reliably be detected and quantified even in limited amounts of formalin-fixed and paraffinembedded (FFPE) tissues. A multitude of studies report on aberrant methylation of immune checkpoint genes, i.e. $P D$ 1, $P D-L 1$, and cytotoxic T-lymphocyte-associated protein 4 (CTLA4) in various malignancies [18-22]. In melanoma, $P D-L 1$ methylation regulates its expression and is associated with melanoma survival [23].

We recently reported on CTLA4 DNA methylation as a potential biomarker predictive for immune checkpoint blockade efficacy [24]. In the present study, we identify methylated CpG (5'-cytosine-phosphate-guanosine-3) loci in the $P D-L 2$ promoter that correlate with mRNA expression in melanoma tissue and cell lines utilizing the The Cancer Genome Atlas (TCGA) cohort and 37 melanoma cell lines. Our survival analyses of our multicenter cohort of 129 melanoma samples prior to anti-PD-1 therapy and the TCGA cohort suggest that PD-L2 DNA methylation might be a prognostic and predictive biomarker in melanoma. These findings are supplemented by recently published mRNA sequencing data of 121 melanoma patients prior to immune checkpoint blockade [25].

\section{Results \\ Promoter methylation of $P D-L 2$ is inversely correlated with mRNA expression}

The Infinium HumanMethylation450 BeadChip contains five beads targeting $\mathrm{CpG}$ sites within the $P D-L 2$ gene locus (Fig. 1). CpG site cg07211259 was located in the promoter region, cg14440664 and cg14351952 were situated in the promoter flanks, and cg14133064 and cg14374994 were located in the gene body.

We analyzed the correlation between methylation of the CpG sites and mRNA expression in $N=468$ melanoma from the TCGA Research Network [26]. We found significant inverse correlations between $P D-L 2$ DNA methylation and mRNA expression levels at two out of five analyzed CpG sites (Table 1). Inverse correlations were strongest at $\operatorname{cg} 07211259$ located in the promoter region and cg14133064. Methylation of the other CpGs within the promoter flanks and the gene body showed a 


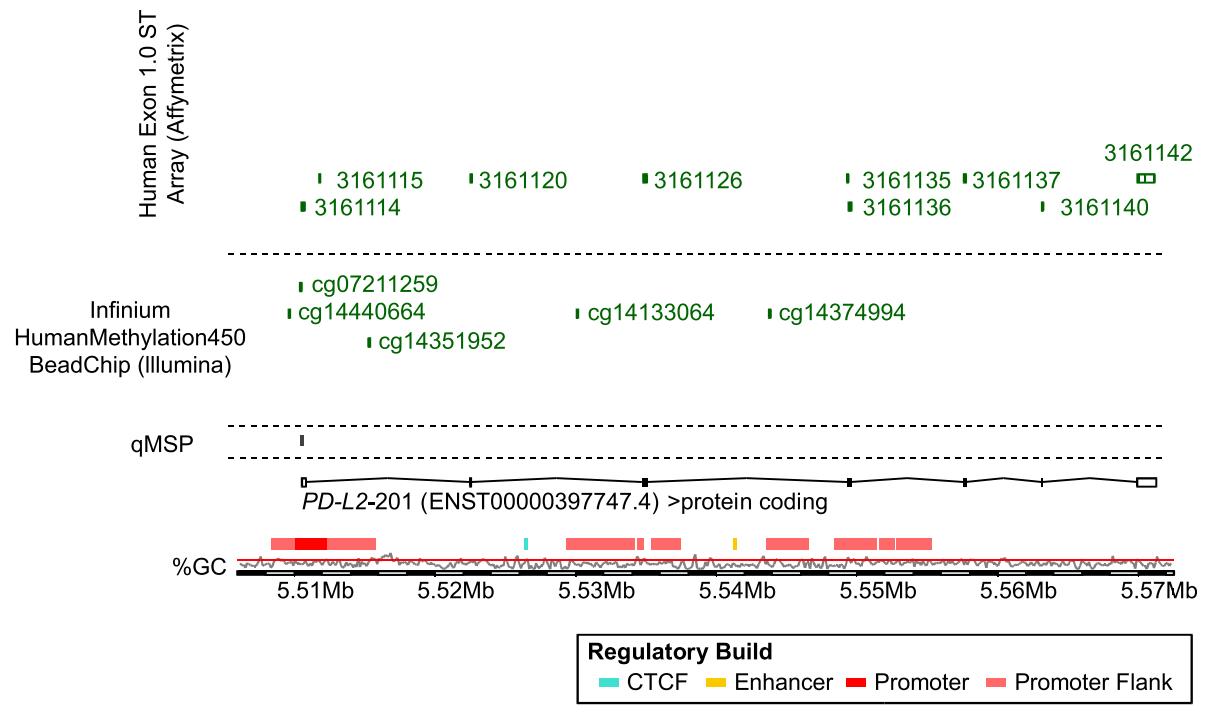

Fig. 1 Genomic organization of the PD-L2 (PDCD1LG2) gene. Shown are regulatory elements, CG density, transcript variants, and target CpG sites of HumanMethylation450 BeadChip beads. The modified illustration was exported from https://www.ensemble.org (Release 95) and is based on Genome Reference Consortium Human Build 38 patch release 12 (GRCh38.p12)

significant positive correlation with mRNA expression. These results suggest that PD-L2 and PD-L1 expression is regulated by gene methylation.

\section{Association of PD-L2 DNA methylation and mRNA expression with patients' survival}

We investigated the relevance of $P D-L 2$ methylation and mRNA expression with patients' overall survival. Methylation and mRNA expression levels were tested as continuous $\log 2$-transformed variates in order to avoid biases due to the introduction of cutoffs for patient sample classification. In univariate Cox proportional analysis, elevated $P D-$ L2 mRNA expression showed a significant correlation with better patients' survival (Hazard ratio $(\mathrm{HR})=0.85$,
95\% CI: 0.77-0.94; Table 1). A positive correlation between elevated methylation levels in the promoter flanks and the gene body (cg14440664, cg14351952, cg14374994) and better patients' survival could be found. In contrast, elevated methylation levels at cg07211259 located in the promoter region were significantly correlated with poor outcome (Table 1). We further dichotomized mRNA levels and methylation levels based on optimized cutoffs for patient classification. Kaplan-Meier survival analyses confirmed better prognosis of patients with high PD-L2 mRNA-expressing (above cutoff) tumors and tumors showing hypomethylation (below cutoff) at cg07211259 located in the promoter region (Fig. 2). In contrast to the $\mathrm{CpG}$ sites in the promoter region,

Table 1 Correlations of PD-L2 methylation with mRNA expression, lymphocyte score, and overall survival

\begin{tabular}{|c|c|c|c|c|c|c|c|}
\hline \multirow[b]{2}{*}{ Analyte } & \multirow{2}{*}{$\begin{array}{l}\text { Methylation or mRNA expression } \\
\text { Mean (95\% confidence interval) }\end{array}$} & \multicolumn{2}{|c|}{$\begin{array}{l}\text { Correlation with mRNA } \\
\text { expression }^{\dagger}\end{array}$} & \multicolumn{2}{|c|}{$\begin{array}{l}\text { Correlation with lymphocyte } \\
\text { score }^{+}\end{array}$} & \multicolumn{2}{|c|}{$\begin{array}{l}\text { Overall survival (Cox proportional } \\
\text { hazards) }\end{array}$} \\
\hline & & Spearman's $\rho$ & $P$ value & Spearman's $\rho$ & $P$ value & Hazard ratio $(95 \%$ Cl) & $P$ value \\
\hline PD-L2 mRNA & $94(79-108)$ & NA & NA & 0.49 & $<0.001$ & $0.85(0.77-0.94)$ & 0.001 \\
\hline $\operatorname{cg} 14440664$ & $72.0(70.6-73.5)$ & 0.11 & 0.018 & 0.10 & 0.078 & $0.63(0.44-0.90)$ & 0.012 \\
\hline cg07211259 & $25.6(23.6-27.5)$ & -0.43 & $<0.001$ & -0.22 & $<0.001$ & $1.16(1.02-1.33)$ & 0.027 \\
\hline cg14351952 & $80.0(78.8-81.1)$ & 0.23 & $<0.001$ & -0.02 & 0.78 & $0.58(0.36-0.92)$ & 0.021 \\
\hline cg14133064 & $51.8(50.1-53.5)$ & -0.18 & $<0.001$ & -0.27 & $<0.001$ & $0.84(0.63-1.12)$ & 0.24 \\
\hline cg14374994 & $86.6(85.9-87.3)$ & 0.32 & $<0.001$ & 0.05 & 0.40 & $0.30(0.10-0.86)$ & 0.025 \\
\hline
\end{tabular}

PD-L2 methylation was determined at five different CpG sites each gene targeted by HumanMethylation450 BeadChip beads (Fig. 1). PD-L2 methylation and mRNA expression were analyzed as log2-transformed variable. Significant features are shown in boldface.

${ }^{\dagger}$ Correlations were performed including $N=468$ (PD-L2 methylation and mRNA expression), $N=328$ (lymphocyte score and PD-L2 $m R N A$ expression), $N=329$ (lymphocyte score and PD-L2 methylation) samples. 




Fig. 2 Overall survival in melanoma patients stratified according to PD-L2 mRNA expression and methylation. Kaplan-Meier analysis of overall survival in melanoma patients stratified according to PD-L2 mRNA expression (left) and methylation levels of the indicated CpG sites. Only CpG sites showing significant survival differences are depicted. Patient samples were dichotomized based on median cutoffs. Follow-up data was available from $N=448$ (mRNA) and $N=450$ (methylation) patients

hypermethylation of cg14440664 located in the promoter flank was associated with better patients' overall survival.

\section{Promoter methylation of $P D-L 2$ is inversely correlated with immune cell infiltrates}

Based on the assumption of a correlation between expression of $P D-L 2$ and adaptive immune cell activity, we tested the correlations between $P D-L 2$ mRNA levels and methylation with lymphocyte score and RNA-Seq signatures of TIL subsets as provided by Thorsson et al. [27]. We found a significant positive association between $P D-L 2$ mRNA expression and lymphocyte score. In accordance, we observed significant inverse associations between PD-L2 methylation and lymphocyte score at $\operatorname{cg} 07211259$ and cg14133064 within the gene promoter and its flank (Table 1). Furthermore, in $P D-L 2$, there were significant inverse correlations between cg07211259 and cg14133064 methylation and mRNA expression signatures of $\mathrm{CD} 4^{+} \mathrm{T}$ cells, regulatory $\mathrm{T}$ cells, activated NK cells, and lymphocytes (Fig. 3).

\section{Promoter methylation of $P D-L 2$ is inversely correlated with an interferon- $\gamma$ signature}

An interferon- $\gamma($ IFN- $\gamma)$ signature has been described as a prognostic and predictive factor in melanoma $[28,29]$.

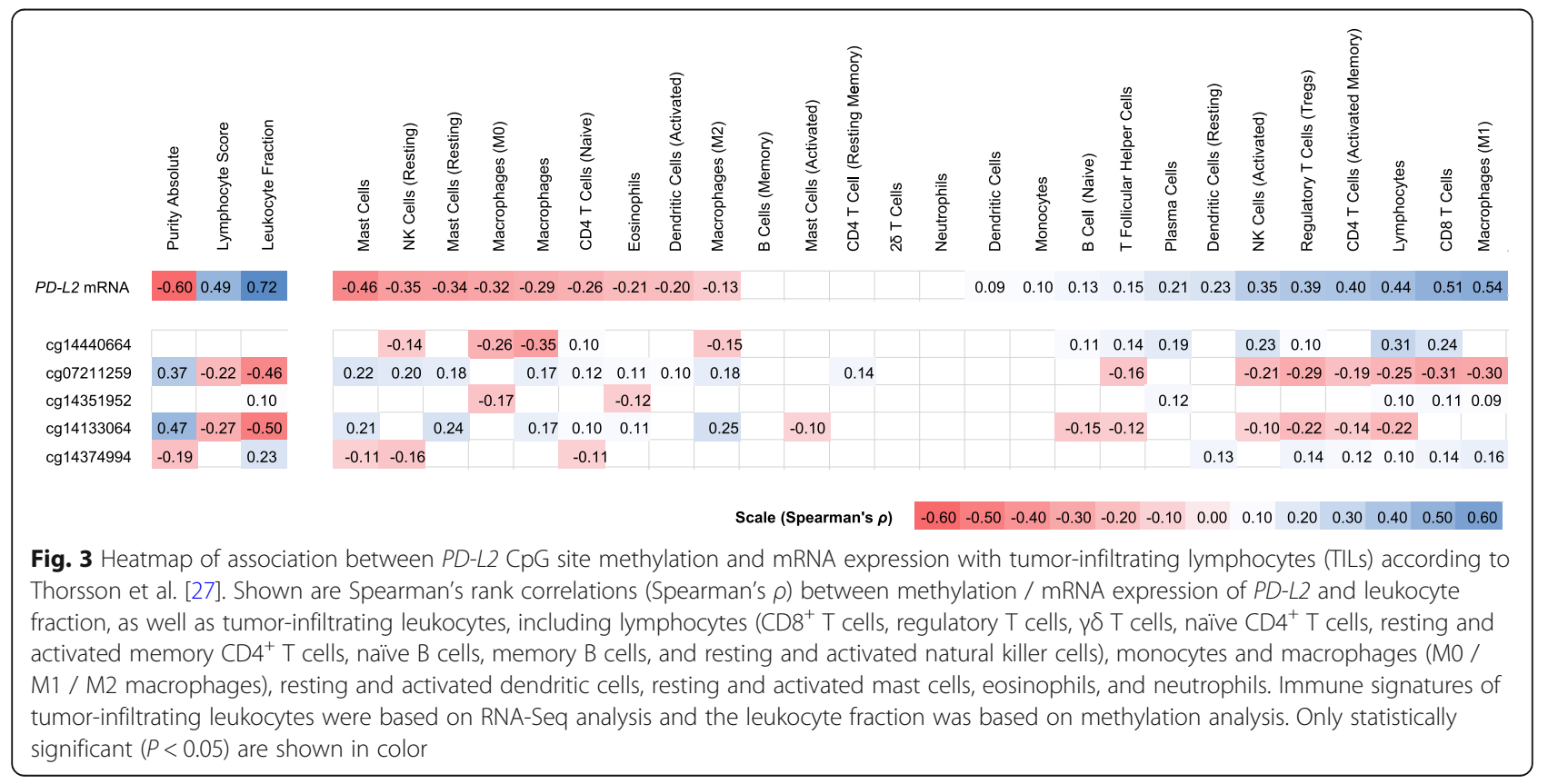


We therefore tested the correlations of $P D-L 2$ methylation and mRNA expression levels with an IFN- $\gamma$ signature defined by the mRNA expression of IFNG itself and IFN- $\gamma$-regulated genes (STAT1, STAT2, STAT3, JAK2, and IRF9; Table 2). PD-L2 mRNA expression levels were significantly correlated with an IFN- $\gamma$ signature. Concordant inverse correlations between $P D-L 2$ methylation levels and IFN- $\gamma$ signature were present predominantly in the promoter region at cg07211259. At cg14133064 in the promoter region, a significant inverse correlation between $P D$ L2 methylation levels and IFNG, STAT1, and IRF9 mRNA expression could be found. The remaining $\mathrm{CpG}$ sites showed positive correlations between methylation level and IFN- $\gamma$ signature and did not significantly correlate with all IFN- $\gamma$-regulated genes.

\section{PD-L2 DNA methylation and mRNA expression in melanoma cell lines}

PD-L2 can be expressed on melanoma cells as well as on tumor-infiltrating immune cells [30, 31]. Therefore, we wanted to test if the correlations between methylation and mRNA expression are simply a measure for the infiltration of PD-L2-expressing immune cells or if tumor cells themselves express PD-L2 epigenetically controlled. We analyzed the correlation between methylation of the CpG sites and mRNA expression in $N=37$ melanoma cell lines [32]. In $P D-L 2$, significant inverse correlation between DNA methylation and mRNA expression was found in the promoter region and its flank at cg07211259 and cg14351952 (Table 3).

\section{Interferon- $\gamma$ induces methylation-dependent PD-L2 protein expression}

In order to analyze the impact of IFN- $\gamma$ on $P D-L 2$ DNA methylation and PD-L2 expression, we examined seven different human melanoma cell lines. Melanoma cell lines were treated with recombinant IFN- $\gamma$ or left untreated for $72 \mathrm{~h}$. Analyses were performed using a quantitative methylation-specific real-time PCR (qMSP) assay targeting CpG site cg07211259. PD-L2 expression was assessed via flow cytometry. 6/7 melanoma cell lines did not show a baseline PD-L2 expression. Only one cell line (SKmel29) displayed 3\% of PD-L2-expressing melanoma cells. Therefore, no correlation could be found for $P D$ L2 DNA methylation and PD-L2 expression in the absence of IFN- $\gamma(\rho=-0.43, P=0.34)$. IFN- $\gamma$ treatment for $72 \mathrm{~h}$ was able to induce PD-L2 expression in 5/7 melanoma cell lines. The two cell lines (BN-SKCM-01 and BN-SKCM-03) that did not respond to IFN- $\gamma$ treatment with PD-L2 upregulation showed a higher $P D-L 2$ promotor methylation than IFN- $\gamma$ responsive melanoma cell lines. IFN- $\gamma$-induced PD-L2 protein expression was significantly correlated with $P D-L 2$ methylation $(\rho=-0.86$, $P=0.014$; Fig. 4a). $P D-L 2$ methylation levels did not significantly alter upon $72 \mathrm{~h}$ of IFN- $\gamma$ treatment (Fig. 4b).

\section{Prognostic and predictive value of $P D-L 2$ DNA methylation and mRNA expression in melanoma samples prior to anti-PD-1 therapy}

To investigate the prognostic and predictive value of $P D-L 2$ DNA methylation, we examined $P D-L 2$ DNA methylation in $N=129$ patients with metastatic melanoma prior to anti-PD-1 immunotherapy. Patient characteristics are shown in Table 4. Analyses were performed using a qMSP assay targeting CpG site cg07211259. We found that $P D-L 2$ DNA methylation dichotomized based on an optimized cutoff $(9.92 \%)$ was significantly correlated with patients' progression-free survival under antiPD-1 immunotherapy $(P=0.023$; Fig. $5 a)$. Patients with low methylation levels showed significant longer

Table 2 Correlations of PD-L2 methylation and mRNA expression with IFN- $\gamma$ signature

\begin{tabular}{|c|c|c|c|c|c|c|c|c|c|c|c|c|}
\hline \multirow[b]{2}{*}{ Analyte } & \multicolumn{2}{|l|}{ IFNG } & \multicolumn{2}{|l|}{ STAT1 } & \multicolumn{2}{|l|}{ STAT2 } & \multicolumn{2}{|l|}{ STAT3 } & \multicolumn{2}{|l|}{ JAK2 } & \multicolumn{2}{|l|}{ IRF9 } \\
\hline & $\begin{array}{l}\text { Spearman's } \\
\rho\end{array}$ & $P$ value & $\begin{array}{l}\text { Spearman's } \\
\rho\end{array}$ & $\begin{array}{l}P \\
\text { value }\end{array}$ & $\begin{array}{l}\text { Spearman's } \\
\rho\end{array}$ & $\begin{array}{l}P \\
\text { value }\end{array}$ & $\begin{array}{l}\text { Spearman's } \\
\rho\end{array}$ & $\begin{array}{l}P \\
\text { value }\end{array}$ & $\begin{array}{l}\text { Spearman's } \\
\rho\end{array}$ & $\begin{array}{l}P \\
\text { value }\end{array}$ & $\begin{array}{l}\text { Spearman's } \\
\rho\end{array}$ & $\begin{array}{l}P \\
\text { value }\end{array}$ \\
\hline $\begin{array}{l}P D-L 2 \\
\text { mRNA }\end{array}$ & 0.81 & $\begin{array}{l}< \\
0.001\end{array}$ & 0.76 & $\begin{array}{l}< \\
0.001\end{array}$ & 0.32 & $\begin{array}{l}< \\
0.001\end{array}$ & 0.36 & $\begin{array}{l}< \\
0.001\end{array}$ & 0.62 & $\begin{array}{l}< \\
0.001\end{array}$ & 0.51 & $\begin{array}{l}< \\
0.001\end{array}$ \\
\hline $\operatorname{cg} 14440664$ & 0.16 & 0.001 & 0.16 & $\begin{array}{l}< \\
0.001\end{array}$ & 0.21 & $\begin{array}{l}< \\
0.001\end{array}$ & 0.04 & 0.45 & 0.14 & 0.002 & 0.12 & 0.008 \\
\hline cg07211259 & -0.46 & $\begin{array}{l}< \\
0.001\end{array}$ & -0.46 & $\begin{array}{l}< \\
0.001\end{array}$ & -0.34 & $\begin{array}{l}< \\
0.001\end{array}$ & -0.13 & 0.004 & -0.21 & $\begin{array}{l}< \\
0.001\end{array}$ & -0.45 & $\begin{array}{l}< \\
0.001\end{array}$ \\
\hline $\operatorname{cg} 14351952$ & 0.13 & 0.004 & 0.19 & $\begin{array}{l}< \\
0.001\end{array}$ & 0.14 & 0.002 & 0.08 & 0.086 & 0.23 & $\begin{array}{l}< \\
0.001\end{array}$ & 0.18 & $\begin{array}{l}< \\
0.001\end{array}$ \\
\hline $\operatorname{cg} 14133064$ & -0.30 & $\begin{array}{l}< \\
0.001\end{array}$ & -0.15 & 0.002 & 0.03 & 0.57 & -0.04 & 0.35 & -0.04 & 0.42 & -0.13 & 0.004 \\
\hline $\operatorname{cg} 14374994$ & 0.24 & $\begin{array}{l}< \\
0.001\end{array}$ & 0.21 & $\begin{array}{l}< \\
0.001\end{array}$ & 0.06 & 0.23 & 0.12 & 0.009 & 0.27 & $\begin{array}{l}< \\
0.001\end{array}$ & 0.12 & 0.007 \\
\hline
\end{tabular}

Correlations of PD-L2 methylation and mRNA expression with mRNA expression of IFNG and IFN- $\gamma$-regulated genes (STAT1, STAT2, STAT3, JAK2, and IRF9). DNA methylation was determined at five different CpG sites, each gene targeted by HumanMethylation450 BeadChip beads (Fig. 1). Significant features are shown in boldface. Data were procurable from $N=468$ tumor samples 
Table 3 PD-L2 methylation and mRNA expression in melanoma cell lines

\begin{tabular}{|c|c|c|c|}
\hline \multirow[b]{2}{*}{ Analyte } & \multirow{2}{*}{$\begin{array}{l}\text { Methylation [\%] or mRNA expression (mRNA: } N=19 \text {, methylation: } N=37 \text { ) } \\
\text { Mean ( } 95 \% \text { confidence interval) }\end{array}$} & \multicolumn{2}{|c|}{ Correlation with mRNA expression $(N=19)$} \\
\hline & & Spearman's $\rho$ & $P$ value \\
\hline$P D-L 2$ mRNA & $6.9(6.4-7.4)$ & NA & NA \\
\hline $\operatorname{cg} 14440664$ & $58.1(49.7-66.5)$ & -0.379 & 0.11 \\
\hline cg07211259 & $26.4(19.3-33.6)$ & -0.504 & 0.028 \\
\hline cg14351952 & $68.5(61.1-75.8)$ & 0.526 & 0.021 \\
\hline cg14133064 & $53.0(44.9-61.2)$ & -0.016 & 0.96 \\
\hline $\operatorname{cg} 14374994$ & $86.2(80.9-91.5)$ & 0.333 & 0.16 \\
\hline
\end{tabular}

$N=37$ melanoma cell lines ( $N=4$ primary melanomas, $N=17$ lymph node metastases, $N=16$ brain metastases) obtained by Marzese et al. [32]. $P D$ - L2 methylation was determined at five different $\mathrm{CpG}$ sites, each gene targeted by HumanMethylation450 BeadChip beads (Fig. 1). Significant features are shown in boldface.

progression-free survival than patients with high methylation levels. We further analyzed RNA-Seq (data provided by Liu et al. [25]) of 121 melanoma patients receiving anti-PD-1 therapy. In accordance with our DNA methylation results, PD-L2 mRNA expression dichotomized based on an optimized cutoff (6.3 Transcripts Per Million (TPM)) was correlated with patients' progression-free survival ( $P=0.030$, Fig. $5 \mathrm{~b})$.

\section{Discussion}

We found significant inverse correlations between PD-L2 methylation and mRNA expression levels at all analyzed promoter CpG sites in melanoma tissue. Thus, our data indicate a gene silencing mechanism via promoter methylation. Significant inverse correlations between PD-L2 methylation and mRNA expression levels at cg07211259 in the promoter region of melanoma cell lines confirmed that melanoma cells themselves express PD-L2 epigenetically controlled. A possible epigenetic regulation of the PD-1 ligands PD-L2 and PD-L1 via DNA methylation has already been postulated for other solid tumors [20, 33, 34]. In melanoma, regulation of PD-L1 expression by DNA promoter methylation has recently been demonstrated in three melanoma cell lines that showed increased PD-L1 expression upon treatment with the hypomethylating agent 5-azacytidine [23]. In our study, we identified significant inverse correlations between PD-L2 DNA methylation and mRNA expression levels in melanoma, that were most pronounced at cg07211259 and cg14133064. CpG site cg07211259 is located in an upstream PD-L2 promoter region. However, according to literature, a second downstream promoter might exist that functions in a lineage-specific fashion and regulates PD-L2 expression in B cells. This downstream promoter appears to be located between exon 1 and exon 2 of the main transcript variant [35]. This may explain the significant inverse correlation between DNA methylation and mRNA expression levels at cg14133064 in the TCGA cohort. Cell type-specific usage of alternative promoters in human genomes has already been described in literature [36]. a

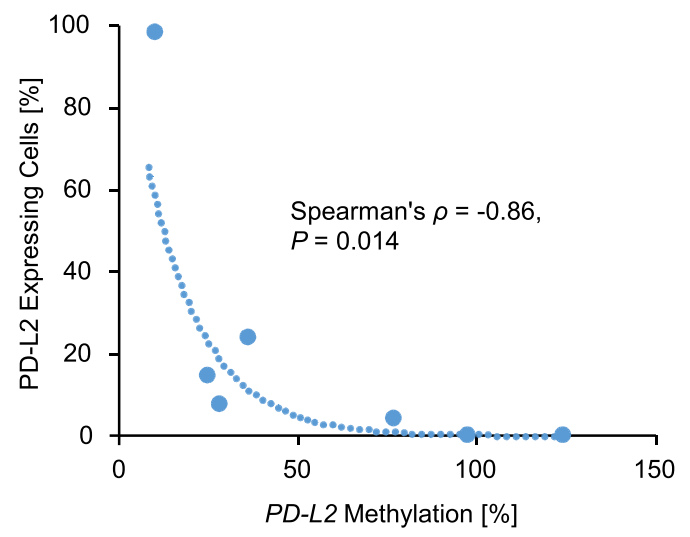

b



Fig. 4 Impact of 72-h IFN- $\gamma$ treatment on PD- $L 2$ methylation and PD- $L 2$ protein expression in human melanoma cell lines. a $P D-L 2$ methylation in correlation to percentage of PD- L2 -expressing melanoma cells upon $72 \mathrm{~h}$ of IFN- $\gamma$ treatment. b PD- $L 2$ methylation of the seven different human melanoma cell lines with or without interferon- $\gamma$ treatment 
Table 4 Patient characteristics of the ICB cohort

\begin{tabular}{|c|c|c|}
\hline Characteristics & Number $N$ & $(\%)$ \\
\hline All patients & 129 & 100 \\
\hline Age in years, (range) & $67.11,(28-92)$ & \\
\hline \multicolumn{3}{|l|}{ Gender } \\
\hline Female & 50 & 38.8 \\
\hline Male & 79 & 61.2 \\
\hline \multicolumn{3}{|l|}{ Site of melanoma metastases } \\
\hline Cutaneous metastases & 62 & 48.1 \\
\hline Lymph node metastases & 37 & 28.7 \\
\hline Lung metastases & 13 & 10.1 \\
\hline Brain metastases & 11 & 8.5 \\
\hline Abdominal metastases & 6 & 4.7 \\
\hline \multicolumn{3}{|l|}{ BRAF mutation status } \\
\hline BRAF wild type & 84 & 65.1 \\
\hline BRAF mutated & 42 & 32.6 \\
\hline Unknown & 3 & 2.3 \\
\hline \multicolumn{3}{|l|}{ Response to anti-PD-1 blockade } \\
\hline Progressive disease (PD) & 58 & 45.0 \\
\hline Partial response (PR) & 39 & 30.2 \\
\hline Stable disease (SD) & 8 & 6.2 \\
\hline Complete response (CR) & 22 & 17.0 \\
\hline Unknown & 2 & 1.6 \\
\hline \multicolumn{3}{|l|}{ Therapeutic regimen } \\
\hline Anti-PD-1 monotherapy & 68 & 52.7 \\
\hline Ipilimumab, anti-PD-1 monotherapy & 12 & 9.3 \\
\hline Ipilimumab + nivolumab & 17 & 13.2 \\
\hline Ipilimumab, ipilimumab + nivolumab & 2 & 1.5 \\
\hline Anti-PD-1 monotherapy, ipilimumab + nivolumab & 30 & 23.3 \\
\hline \multicolumn{3}{|l|}{ Medical center } \\
\hline University Hospital Bonn—dermatology & 104 & 80.6 \\
\hline University Hospital Bonn—oncology & 5 & 3.9 \\
\hline University Hospital Bonn—neurosurgery/-oncology & 4 & 3.1 \\
\hline Kantonsspital St. Gallen, Spital Grabs, Spital Wil, Spital Flawil & 16 & 12.4 \\
\hline
\end{tabular}

Data include age, gender, site of the melanoma metastases, BRAF mutation status, response to anti-PD-1 blockade, therapeutic regimen, and the treating medical center of $N=129$ stage IV melanoma patients receiving immune checkpoint blockade

We assume that this second downstream promoter might only be relevant in B cells, but not in melanoma cells. This would explain the insignificant correlation between cg14133064 methylation level and mRNA expression levels in melanoma cell lines.

A positive correlation between $P D-L 2$ mRNA expression and $P D-L 2$ DNA methylation could be detected at the remaining CPG sites located in the promoter flanks. Such extensive positive correlations between methylation and transcriptional activity are frequently found in gene bodies [37]. In conclusion, our data suggest that in accordance to $P D-L 1, P D-L 2$
mRNA expression is regulated by promoter methylation in melanoma.

Further experiments are needed to establish a causal relationship between DNA methylation of the promoter region and PD-L2 transcription. In our study, we refrained from using demethylating agents as for example 5-aza-2deoxycytidine, as it does not specifically demethylate $P D$ $L 2$, but instead leads to an unspecific demethylation of the whole genome which may also include transcription factors and cytokines. A more specific and elegant approach could be a specific zinc finger-induced methylation of the $P D-L 2$ promoter, as it has been described by $\mathrm{Li}$ et al. in 



Fig. 5 Progression-free survival in two cohorts of metastasized melanoma patients treated with immune checkpoint blockade in association to PD-L2 methylation and PD-L2 mRNA expression. Kaplan-Meier analysis of progression-free survival in metastasized melanoma patients stratified according to cg07211259 methylation (a) and PD-L2 mRNA expression (b) levels in pre-treatment samples. Patient samples were dichotomized based on optimized cutoffs. Follow-up data was available from $N=129$ (methylation cohort) and $N=121$ (mRNA expression cohort obtained from Liu et al. [25]) patients, respectively

the context of $P D-L 1$ [38]. However, until now, this is not an established method.

The prognostic value of PD-L2/PD-L1 expression in melanoma is still unclear. PD-L1 protein expression of melanoma cells detected by IHC has been described as an independent prognostic factor for melanoma [39, 40]. Obeid et al. demonstrated that in 147 metastatic melanomas, PD-L1 and PD-L2 expression defined by positive IHC on tumor cells is associated with improved overall survival [7]. However, PD-L1 expression has also been reported to be correlated with an unfavorable prognosis in various malignancies, including melanoma [41]. In our study, PD-L2 DNA promoter hypomethylation and high mRNA expression were found to be a predictor of prolonged overall survival in the absence of anti-PD-1 immunotherapy. In line with these results, we found $P D-L 2$ DNA methylation and mRNA expression to be correlated with known prognostic factors, such as tumor-infiltrating lymphocytes and an IFN- $\gamma$ signature. Tumor-infiltrating lymphocytes are known to be associated with favorable prognosis in primary and advanced melanoma [42, 43]. An IFN- $\gamma$ signature has been shown to be associated with response to immune checkpoint inhibitors [44, 45]. Thus, $P D-L 2$ DNA promoter hypomethylation and high mRNA expression might be prognostic factors, associated with improved overall survival in melanoma patients.

In order to further analyze the correlation between $P D$-L2 DNA methylation and PD-L2 expression, we examined PD-L2 expression in human melanoma cell lines with and without IFN- $\gamma$ treatment. As expected, treatment with IFN- $\gamma$ did not change DNA methylation levels, but did increase PD-L2 expression.
Hypermethylated melanoma cell lines showed a significantly lower PD-L2 expression after IFN- $\gamma$ treatment than hypomethylated cell lines. This supports our hypothesis, that IFN- $\gamma$-induced PD-L2 expression in melanoma cells is controlled by the degree of DNA methylation of the PD-L2 promoter region. Of note, we did not find a correlation between PD-L2 protein expression and $P D-L 2$ promoter methylation in the absence of IFN- $\gamma$. This fact might point towards the utility of $P D-L 2$ methylation testing to determine not only the effective, potentially transient PD-L2 protein expression but also the general ability of melanoma cells to express PD-L2 protein under specific proinflammatory conditions. This finding would suggest a higher biomarker performance of PD-L2 methylation compared to PD-L2 expression.

To increase therapeutic efficacy and reduce treatmentrelated morbidity, predictive and prognostic biomarkers are urgently needed to identify patients that are most likely to benefit from checkpoint blockade. PD-L1 protein expression measured by IHC has been shown to be associated with response to anti-PD-1 therapy in certain studies, but its performance as predictive biomarker remains insufficient [12]. Our data suggest that PD-L2 DNA promoter hypomethylation might be correlated with patients' prolonged progression-free survival under anti-PD-1 antibody therapy. Consistent with our results obtained from the DNA methylation analysis, high $P D$ L2 mRNA expression appears to be correlated with patients' prolonged progression-free survival in metastatic melanoma patients under anti-PD-1 therapy provided by Liu et al. [25]. Thus, PD-L2 DNA methylation and 
mRNA expression seem to be predictive biomarkers in melanoma patients receiving anti-PD-1 therapy.

Our study covers only a limited number of $\mathrm{CpG}$ sites within $P D-L 2$. Hence, further studies, i.e., using bisulfite sequencing, should be performed to identify the CpG sites showing the highest biomarker performance. A special focus should be placed on CpG sites in enhancers and potential alternative promoters. As our analyzed CpGs sites already show a correlation of DNA methylation levels with mRNA expression and overall survival of melanoma patients, it is possible that other CpG sites will exhibit an even better biomarker performance.

We are aware of the limitations of our present study. The analysis of multiple CpG sites is, in general, vulnerable to multiple testing issues. Results would therefore have to be validated in an independent cohort. In order to reduce the risk of multiple testing artifacts, we reported the unselected results for all CpG sites analyzed in our study. Furthermore, in our patient cohort receiving anti-PD-1 therapy, data were dichotomized based on optimized cutoffs. When dichotomized based on median cutoffs, significant results could not be found, which might be due to the small sample size of only 129 or 121 melanoma patients, respectively. Therefore, we cannot refer $P D-L 2$ as a definite predictive biomarker and further validation is required.

\section{Conclusion}

Biomarkers that allow for the prediction of clinical response to anti-PD-1 therapy are desperately needed. Our data suggest an epigenetic regulation of PD-L2 expression via DNA methylation and a predictive value for progression-free survival in anti-PD-1 treated melanoma patients. We conclude that in contrast to PD-L2 protein expression, $P D-L 2$ methylation allows to determine not only the effective expression status of melanoma cells, but also the ability to express PD-L2 under proinflammatory conditions, i.e., in the presence of IFN- $\gamma$. Assessment of $P D-L 2$ promoter methylation and expression testing therefore should be included in further clinical trials with anti-PD-1 antibodies.

\section{Material and methods \\ Patients}

\section{TCGA cohort}

The data we analyzed are partly based on datasets from The Cancer Genome Atlas Research Network (TCGA, http://cancergenome.nih.gov/). We included $N=470$ primary solid and metastatic melanoma tumor tissue samples from the TCGA skin cutaneous melanoma (SKCM) cohort. The TCGA Research Network obtained informed consent from all patients in accordance with the Helsinki Declaration of 1975 . One sample per patient was analyzed. In patients providing more than one sample, metastatic tumor samples were included. We used the TCGA Research Network to obtain supplementary clinicopathological data. Molecular data were adopted from a study previously published by the TCGA Research Network [26]. Datasets comprising information about sample purity and ploidy estimates were adopted from the TCGA Research Network and calculated using the ABSOLUTE algorithm [46]. We used the results provided by Thorsson at al [27]. who developed RNA sequencing (RNA-Seq) signatures as a surrogate for immune cell infiltrates to obtain quantitative data on infiltrating leukocytes. Data on infiltrating lymphocytes were adopted from the TCGA Research Network [26], including lymphocyte distribution (03; $0=$ no lymphocytes within the tissue, $1=$ lymphocytes present involving $<25 \%$ of the tissue cross-sectional area, $2=$ lymphocytes present in 25 to $50 \%$ of the tissue, $3=$ lymphocytes present in $>50 \%$ of tissue), lymphocyte density $(0-3 ; 0=$ absent, $1=$ mild, $2=$ moderate, $3=$ severe $)$, and lymphocyte score $(0-6$, score defined as the sum of the lymphocyte distribution and density scores).

\section{ICB melanoma cohort}

Patients $(N=129)$ diagnosed with metastatic melanoma and treated with anti-PD-1 checkpoint inhibition at the University Hospital Bonn, the Kantonsspital St. Gallen, the Spital Grabs, the Spital Wil, and Spital Flawil between May 2012 and June 2019 were included in the cohort (patients characteristics are shown in Table 4). Response patterns were reported based on Response Evaluation Criteria in Solid Tumors (RECIST). The study protocol was approved by the Institutional Review Board.

\section{Patient cohort provided by Liu et al. [25]}

Advanced melanoma patients $(N=121)$ treated with an anti-PD-1 antibody alone or in combination with an anti-CLTA-4 antibody in a palliative setting were included in the cohort. Response patterns were reported based on RECIST criteria.

\section{Melanoma cell lines}

We included $N=37$ melanoma cell lines $(N=4$ primary melanomas, $N=17$ lymph node metastases, $N=16$ brain metastases) obtained by Marzese et al. [32] (Gene Expression Omnibus (GEO) accession number: GSE44662; National Center for Biotechnology Information (NCBI), Bethesda, MD, USA). Furthermore, we included seven human melanoma cell lines (BN-SKCM-01, BN-SKCM-02, BN-SKCM-03, A375, MaMel85, SKmel28, SKmel29). The MaMel85 human melanoma cell line were established, characterized and kindly provided by Dirk Schadendorf (University Hospital Essen, Essen, Germany). BN-SKCM01, BN-SKCM-02, and BN-SKCM-03 were originally isolated from melanoma metastases collected from adult 
donors treated at the University Hospital Bonn with participants' informed consent. All human cells were cultured in complete RPMI 1640 medium supplemented with 10\% FCS (Merck KGaA, Darmstadt, Germany), $2 \mathrm{mM}$ Lglutamine (Life Technologies, Carlsbad, CA, USA), 10 $\mathrm{mM}$ non-essential amino acids (Life Technologies), $1 \mathrm{mM}$ HEPES (Life Technologies), $20 \mathrm{mM}$ 2-mercaptoethanol, $100 \mathrm{U} / \mathrm{ml}$ penicillin, and $100 \mathrm{mg} / \mathrm{ml}$ streptomycin (Life Technologies). Melanoma cell lines were either left untreated over $72 \mathrm{~h}$ or treated with recombinant IFN- $\gamma$ (1000 U/ml IFN- $\gamma$, PeproTech, Rocky Hill, NJ, USA).

\section{mRNA expression analysis TCGA cohort}

The mRNA expression data was generated using the Illumina HiSeq 2000 RNA Sequencing Version 2 analysis (Illumina, Inc., San Diego, CA, USA). Expression data of level 3 were provided by the TCGA Research Network. Data were available from $N=468$ patient samples. Normalized counts (n.c.) per genes were calculated using the SeqWare framework via the RSEM (RNA-Seq by Expectation Maximization) algorithm [47].

\section{Patient cohort provided by Liu et al.}

Liu and colleagues performed the extraction of mRNA from $N=121$ FFPE melanoma tissue obtained before anti-PD-1 therapy using the QIAGEN AllPrep DNA/ RNA Mini Kit (Qiagen, Hilden, Germany). RNA sequencing was performed using the Illumina sequencing platform and TPM levels were reported [25].

\section{Melanoma cell lines}

Whole-transcript expression data generated with the $\mathrm{Hu}-$ man Exon 1.0 ST Array (Affymetrix, Inc., Santa Clara, CA, USA) was obtained from $N=19$ melanoma cell lines $(N=$ 12 lymph node metastases, $N=7$ brain metastases; GSE44662) [32]. We used mean values of the probe sets 3161114, 3161115, 3161120, 3161126, 3161135, 3161136, 3161137, 3161140, and 3161142 (Fig. 1).

\section{Methylation analysis}

Data on gene methylation ( $\beta$-values) were obtained from the TCGA Research Network and downloaded from the UCSC Xena browser (https://www.xena.ucsc.edu). Data were available from $N=470$ patient samples. In addition, $\beta$-values of melanoma cell lines were downloaded from Marzese et al. (GSE44662) [32]. Both methylation studies were performed using the Infinium HumanMethylation450 BeadChip (Illumina, Inc.).

We performed qMSP analysis of additional melanoma cell lines and FFPE melanoma tissues using bisulfiteconverted DNA prepared by means of the innuCONVERT Bisulfite All-In-One Kit (Analytik Jena, Jena, Germany) according to the manufacturer's instructions.
Prior to bisulfite conversion, we macrodissected tumor areals from FFPE melanoma tissue sections mounted on glass slides. Our $P D-L 2$ qMSP assay was duplexed with an $A C T B$ assay in order to quantify the total amount of DNA. We used a $100 \%$ methylated calibrator sample (CpGenome $^{\mathrm{TM}}$ Universal Methylated DNA, Millipore, MA, USA) for the calibration of the qMSP results. qMSP reactions were performed in $20 \mu \mathrm{l}$ volumes containing 20 ng bisulfite-converted sample and calibrator DNA (quantified via UV-VIS spectrophotometry). We performed triplicate measurements of each sample and the calibrator. PCR buffer conditions were used as previously described [48]. Real-time PCR was carried out using a 7900HT Fast Real-Time PCR system (Applied Biosystems, Waltham, MA, USA) applying the following temperature profile: 20 min at $95^{\circ} \mathrm{C}$ and 40 cycles with $2 \mathrm{~s}$ at $62^{\circ} \mathrm{C}, 60 \mathrm{~s}$ at $56^{\circ} \mathrm{C}$ (each at $100 \%$ ramp rate), and $15 \mathrm{~s}$ at $95^{\circ} \mathrm{C}$ (at $75 \%$ ramp rate). Percentage methylation levels were calculated using cycle threshold (CT) values according to the $\Delta \Delta C T$ method as described before [48]. The following oligonucleotides and final concentrations per PCR reaction were used: $\quad A C T B$ probe: ATTO $647 \mathrm{~N}$ accaccacccaacacacaataacaaacaca-BHQ-2 $\quad(0.125 \mu \mathrm{M})$; $A C T B$ forward primer: gtgatggaggaggtttagtaagtt $(0.125 \mu \mathrm{M})$; ACTB reverse primer: ccaataaaacctactcctcccttaa $(0.125 \mu \mathrm{M}) ; P D-L 2$ probe: 6-FAM-ttatttttatgttacggtaaattttaa-BHQ-1 $(0.4 \mu \mathrm{M}) ; \quad P D-L 2$ forward primer: aaaatttttaaataagttaggttttc $(0.3 \mu \mathrm{M})$; and $P D-L 2$ reverse primer: caaaaaaacactcaaaatttaacgt $(0.3 \mu \mathrm{M})$.

\section{Flow cytometry}

Melanoma cells were stained with the following antibodies according to standard procedures: fluorochromeconjugated monoclonal antibody specific for human PDL2 (Clone 24F.10C12, BioLegend, San Diego, CA, USA). Apoptosis induction and cell cycle arrest were analyzed using FITC Annexin V Apoptosis Detection Kit (BioLegend). Data were acquired with a FACSCanto flow cytometer (BD Biosciences, San Jose, CA, USA) and analyzed with FlowJo software (V7.6.5 for Windows, TreeStar, Ashland, OR, USA).

\section{Statistics}

Statistical analyses were performed using SPSS, version 23.0 (SPSS Inc., Chicago, IL, USA). Analyses regarding potential correlations of characteristics were calculated using Spearman's rank correlation (Spearman's $\rho$ ). Mean value comparisons were performed using WilcoxonMann-Whitney $U$ (two groups) and Kruskal-Wallis ( $>2$ groups) test. One-way ANOVA and post-hoc Bonferroni test were applied to perform multiple comparisons between groups. In order to reduce the influence of agerelated deaths, survival was censored after 5 years (1825 days). Kaplan-Meier method and Cox proportional 
hazards regression were used for the performance of survival analyses. Progression-free survival (PFS) was defined as the time between the first application of antiPD-1 antibody and the date of documented disease progression. Overall survival (OS) was defined as time between initial diagnosis and death or last contact, respectively. For Kaplan-Meier analysis methylation levels and mRNA expression levels were dichotomized based on an optimized cutoff (lowest $P$ value). Cox proportional hazards analyses were performed with log2transformed methylation and mRNA expression data (mRNA expression levels of 0 n.c. were set to 0.1 prior to $\log 2$-transformation). $P$ values refer to log-rank and Wald tests. Two-sided $P$ values less than 0.05 were considered statistically significant.

\begin{abstract}
Abbreviations
95\% Cl: 95\% Confidence interval; B2M: Beta-2 microglobulin; CpG: 5'cytosine-phosphate-guanosine-3'; CR: Complete response; CTLA4: Cytotoxic T-lymphocyte-associated protein 4; FFPE: Formalin-fixed and paraffinembedded; HR: Hazard ratio; IHC: Immunohistochemistry; IFN- $\gamma$, IFNG: Interferon gamma; JAK: Janus kinase; mRNA: Messenger ribonucleic acid; NA: Not applicable; NK cells: Natural killer cells; OS: Overall survival; PCR: Polymerase chain reaction; PD: Progressive disease; PD-1: Programmed cell death 1; PD-L1: Programmed cell death 1 ligand 1; PD-L2: Programmed cell death 1 ligand 2; PFS: Progression-free survival; PR: Partial response; GMSP: Quantitative methylation-specific PCR; RNA-Seq: RNA sequencing; SD: Stable disease; STAT: Signal transducer and activator of transcription; TCGA: The Cancer Genome Atlas; TILs: Tumor-infiltrating lymphocytes; TPM: Transcripts per million
\end{abstract}

\section{Acknowledgements}

We thank all members of the Landsberg and Dietrich lab for critical discussions. We thank Dirk Schadendorf and the University Hospital Essen UKE (Germany) for providing human melanoma cell lines. We thank the BioBank Bonn of the Bonn University Medical Faculty and the University Hospital Bonn for the support of this study.

\section{Authors' contributions}

$F H, D D$, and $J L$ were involved in the study design and concept. RZ, GS, PK, and AF performed the experiments. FH, JL, and DD drafted the manuscript. $D D$ and JD performed the statistical analyses. RZ, DN, CP, JS, AF, TAWH, GG, SSR, SS, LF, and GK revised the manuscript for critical intellectual content. $D N, G G, G K, P B, T A W H, S S, T P, C P$, and SSR provided patient samples. All authors read and approved the final version of the manuscript.

\section{Funding}

LF is supported by grant PP00P3_157448 from the Swiss National Science Foundation. AF was partly funded by the Mildred Scheel Foundation. DN was funded in part by DFG Cluster of Excellence ImmunoSensation (EXC 1023).

\section{Availability of data and materials}

The datasets generated during and/or analyzed during the current study are available from the corresponding author on reasonable request.

\section{Ethics approval and consent to participate}

Data generation by the TCGA Research Network and Liu et al. was performed in accordance with the Helsinki Declaration of 1975 as previously described $[25,26]$. Patient inclusion and sample analyses at the University Hospital Bonn were approved by the Institutional Review Board (IRB) of the University Hospital Bonn (vote 187/16). Patient inclusion and sample analyses at the Kantonsspital St. Gallen, the Spital Grabs, the Spital Wil, and Spital Flawil were approved by EKOS (Ethikkommission Ostschweiz, Switzerland). Informed consent was obtained from all individual participants included in the study.

\section{Consent for publication}

Not applicable

\section{Competing interests}

DD owns patents on methylation of immune checkpoint genes (including PD-L2) as predictive and prognostic biomarkers. The patents are licensed to Qiagen $\mathrm{GmbH}$ (Hilden, Germany), and DD receives royalty payments. The University Hospital Bonn (PI Dimo Dietrich) received research funding from Qiagen. DD is a consultant for AJ Innuscreen GmbH (Berlin, Germany), a $100 \%$ daughter company of Analytik Jena AG (Jena, Germany), and receives royalties from product sales (innuCONVERT kits). AF, CP, DN, FH, and JS have received speakers' honoraria or travel expense reimbursements from the following companies: Celgene, Roche, BMS, Pierre Fabre, Novartis, and MSD. JL and CP are consultant / advisory board members of Bristol-Myers Squibb, Merck, Novartis, and Roche. LF reported grants from the Swiss National Science Foundation, Hookipa Pharma, Krebsliga Schweiz, and Novartis Foundation as well as an advisory role for Novartis, Sanofi, and Bristol-Myers Squibb. No potential conflicts of interest were disclosed by the other authors.

\section{Author details}

'Department of Dermatology and Allergology, University Hospital Bonn, Bonn, Germany. ${ }^{2}$ Department of Otolaryngology, Head and Neck Surgery, University Hospital Bonn, Venusberg-Campus 1, 53127 Bonn, Germany. ${ }^{3}$ Department of Dermatology and Allergology, Technical University of Munich, Munich, Germany. ${ }^{4}$ Faculty of Medicine, Sigmund Freud University, Vienna, Austria. ${ }^{5}$ Department of Oncology, Hematology and Rheumatology, University Hospital Bonn, Bonn, Germany. ${ }^{6}$ Institute of Neuropathology, University Hospital Bonn, Bonn, Germany. "Microbiology and Immunology PhD Program, University of Zurich, Zurich, Switzerland. ${ }^{8}$ Institute of Immunobiology, Kantonsspital St Gallen, St Gallen, Switzerland. ${ }^{9}$ Department of Oncology and Hematology, Kantonsspital St Gallen, St Gallen, Switzerland.

${ }^{10}$ Department of Dermatology, University Hospital Zurich, Zurich, Switzerland. ${ }^{11}$ Department of Dermatology and Allergology, Kantonsspital St Gallen, St Gallen, Switzerland. ${ }^{12}$ Institute of Pathology, University Hospital Bonn, Bonn, Germany.

Received: 13 March 2020 Accepted: 10 June 2020

Published online: 26 June 2020

\section{References}

1. Eggermont AMM, Blank CU, Mandala M, Long GV, Atkinson V, Dalle S, et al. Adjuvant pembrolizumab versus placebo in resected stage III melanoma. N Engl J Med. 2018 May 10;378(19):1789-801.

2. Weber J, Mandala M, Del Vecchio M, Gogas HJ, Arance AM, Cowey CL, et al. Adjuvant nivolumab versus ipilimumab in resected stage III or IV melanoma. N Engl J Med. 2017;377(19):1824-35.

3. Tseng SY, Otsuji M, Gorski K, Huang X, Slansky JE, Pai SI, et al. B7-DC, a new dendritic cell molecule with potent costimulatory properties for T cells. J Exp Med. 2001 Apr 2;193(7):839-46.

4. Youngnak P, Kozono Y, Kozono H, Iwai H, Otsuki N, Jin H, et al. Differential binding properties of $\mathrm{B} 7-\mathrm{H} 1$ and $\mathrm{B} 7-\mathrm{DC}$ to programmed death-1. Biochem Biophys Res Commun. 2003 Aug 1;307(3):672-7.

5. Messal N, Serriari N-E, Pastor S, Nunès JA, Olive D. PD-L2 is expressed on activated human T cells and regulates their function. Mol Immunol. 2011 Sep;48(15-16):2214-9.

6. Zhong X, Tumang JR, Gao W, Bai C, Rothstein TL. PD-L2 expression extends beyond dendritic cells/macrophages to B1 cells enriched for $\mathrm{V}(\mathrm{H}) 11 \mathrm{~N}(\mathrm{H}) 12$ and phosphatidylcholine binding. Eur J Immunol. 2007 Sep;37(9):2405-10.

7. Obeid JM, Erdag G, Smolkin ME, Deacon DH, Patterson JW, Chen L, et al. PD-L1, PDL2 and PD-1 expression in metastatic melanoma: correlation with tumor-infiltrating immune cells and clinical outcome. Oncoimmunology. 2016;5(11):e1235107.

8. Yearley JH, Gibson C, Yu N, Moon C, Murphy E, Juco J, et al. PD-L2 Expression in human tumors: relevance to anti-PD-1 therapy in cancer. Clin Cancer Res. 2017 Jun 15;23(12):3158-67.

9. Taube JM, Klein A, Brahmer JR, Xu H, Pan X, Kim JH, et al. Association of PD1, PD-1 ligands, and other features of the tumor immune microenvironment with response to anti-PD-1 therapy. Clin Cancer Res. 2014 Oct 1;20(19):5064-74.

10. Topalian SL, Hodi FS, Brahmer JR, Gettinger SN, Smith DC, McDermott DF, et al. Safety, activity, and immune correlates of anti-PD-1 antibody in cancer. N Engl J Med. 2012 Jun 28;366(26):2443-54. 
11. Lee H-H, Wang Y-N, Xia W, Chen C-H, Rau K-M, Ye L, et al. Removal of Nlinked glycosylation enhances PD-L1 detection and predicts anti-PD-1/PDL1 therapeutic efficacy. Cancer cell. 2019 Aug 12;36(2):168-178.e4.

12. Havel JJ, Chowell D, Chan TA. The evolving landscape of biomarkers for checkpoint inhibitor immunotherapy. Nat Rev Cancer. 2019;19(3):133-50.

13. Herbst RS, Soria J-C, Kowanetz M, Fine GD, Hamid O, Gordon MS, et al. Predictive correlates of response to the anti-PD-L1 antibody MPDL3280A in cancer patients. Nature. 2014;515(7528):563-7.

14. Durek P, Nordström K, Gasparoni G, Salhab A, Kressler C, de Almeida M, et al. Epigenomic profiling of human CD4+ T cells supports a linear differentiation model and highlights molecular regulators of memory development. Immunity. 2016;45(5):1148-61.

15. Ghoneim HE, Fan Y, Moustaki A, Abdelsamed HA, Dash P, Dogra P, et al. De novo epigenetic programs inhibit PD-1 blockade-mediated T cell rejuvenation. Cell. 2017 Jun 29;170(1):142-157.e19.

16. Scharer CD, Barwick BG, Youngblood BA, Ahmed R, Boss JM. Global DNA methylation remodeling accompanies CD8 T cell effector function. J Immunol. 2013 Sep 15;191(6):3419-29.

17. Egger G, Liang G, Aparicio A, Jones PA. Epigenetics in human disease and prospects for epigenetic therapy. Nature. 2004 May 27;429(6990):457-63.

18. Chen Y-P, Zhang J, Wang Y-Q, Liu N, He Q-M, Yang X-J, et al. The immune molecular landscape of the B7 and TNFR immunoregulatory ligand-receptor families in head and neck cancer: a comprehensive overview and the immunotherapeutic implications. Oncoimmunology. 2017;6(3):e1288329.

19. Gevensleben H, Holmes EE, Goltz D, Dietrich J, Sailer V, Ellinger J, et al. PDL1 promoter methylation is a prognostic biomarker for biochemical recurrence-free survival in prostate cancer patients following radical prostatectomy. Oncotarget. 2016 Nov 29;7(48):79943-55.

20. Goltz D, Gevensleben H, Grünen S, Dietrich J, Kristiansen G, Landsberg J, et al. PD-L1 (CD274) promoter methylation predicts survival in patients with acute myeloid leukemia. Leukemia. 2017;31(3):738-43.

21. Goltz D, Gevensleben H, Dietrich J, Ellinger J, Landsberg J, Kristiansen G, et al. Promoter methylation of the immune checkpoint receptor PD-1 (PDCD1) is an independent prognostic biomarker for biochemical recurrence-free survival in prostate cancer patients following radical prostatectomy. Oncoimmunology. 2016:5(10):e1221555.

22. Röver LK, Gevensleben H, Dietrich J, Bootz F, Landsberg J, Goltz D, et al. PD1 (PDCD1) promoter methylation is a prognostic factor in patients with diffuse lower-grade gliomas harboring isocitrate dehydrogenase (IDH) mutations. EBioMedicine. 2018 Feb;28:97-104.

23. Micevic G, Thakral D, McGeary M, Bosenberg MW. PD-L1 methylation regulates PD-L1 expression and is associated with melanoma survival. Pigment Cell Melanoma Res. 2019;32(3):435-40.

24. Goltz D, Gevensleben H, Vogt TJ, Dietrich J, Golletz C, Bootz F, et al. CTLA4 methylation predicts response to anti-PD-1 and anti-CTLA-4 immunotherapy in melanoma patients. JCI Insight. 2018;3(13):e96793.

25. Liu D, Schilling B, Liu D, Sucker A, Livingstone $E$, Jerby-Amon $L$, et al. Integrative molecular and clinical modeling of clinical outcomes to PD1 blockade in patients with metastatic melanoma. Nat Med. 2019 Dec;25(12): 1916-27.

26. The Cancer Genome Atlas Network. Genomic classification of cutaneous melanoma. Cell. 2015;161(7):1681-96.

27. Thorsson V, Gibbs DL, Brown SD, Wolf D, Bortone DS, Ou Yang T-H, et al. The immune landscape of cancer. Immunity. 2018;48(4):812-830.e14.

28. Dizier B, Callegaro A, Debois M, Dreno B, Hersey P, Gogas HJ, et al. A Th1/ IFNy gene signature is prognostic in the adjuvant setting of resectable high-risk melanoma but not in non-small cell lung cancer. Clin Cancer Res. 2020 Apr 1;26(7):1725-35.

29. Ribas A, Robert C, Hodi FS, Wolchok JD, Joshua AM, Hwu W-J, et al. Association of response to programmed death receptor 1 (PD-1) blockade with pembrolizumab (MK-3475) with an interferon-inflammatory immune gene signature. J Clin Oncol. 2015;33(15_suppl):3001.

30. Lau J, Cheung J, Navarro A, Lianoglou S, Haley B, Totpal K, et al. Tumour and host cell PD-L1 is required to mediate suppression of anti-tumour immunity in mice. Nat Commun. 2017:8:14572

31. Noguchi T, Ward JP, Gubin MM, Arthur CD, Lee SH, Hundal J, et al. Temporally distinct PD-L1 expression by tumor and host cells contributes to immune escape. Cancer Immunol Res. 2017;5(2):106-17.

32. Marzese DM, Scolyer RA, Huynh JL, Huang SK, Hirose H, Chong KK, et al. Epigenome-wide DNA methylation landscape of melanoma progression to brain metastasis reveals aberrations on homeobox $D$ cluster associated with prognosis. Hum Mol Genet. 2014 Jan 1;23(1):226-38.

33. Franzen A, Vogt TJ, Müller T, Dietrich J, Schröck A, Golletz C, et al. PD-L1 (CD274) and PD-L2 (PDCD1LG2) promoter methylation is associated with HPV infection and transcriptional repression in head and neck squamous cell carcinomas. Oncotarget. 2018 Jan 2;9(1):641-50.

34. Lingohr P, Dohmen J, Semaan A, Branchi V, Dietrich J, Bootz F, et al. Clinicopathological, immune and molecular correlates of PD-L2 methylation in gastric adenocarcinomas. Epigenomics. 2019;11(6):639-53.

35. Kaku H, Rothstein TL. Octamer binding protein 2 (Oct2) regulates PD-L2 gene expression in B-1 cells through lineage-specific activity of a unique, intronic promoter. Genes Immun. 2010 Jan;11(1):55-66.

36. Landry J-R, Mager DL, Wilhelm BT. Complex controls: the role of alternative promoters in mammalian genomes. Trends Genet. 2003 Nov;19(11):640-8.

37. Jones PA. Functions of DNA methylation: islands, start sites, gene bodies and beyond. Nat Rev Genet. 2012 May 29;13(7):484-92.

38. Li X, Wang Z, Huang J, Luo H, Zhu S, Yi H, et al. Specific zinc finger-induced methylation of PD-L1 promoter inhibits its expression. FEBS Open Bio. 2019; 9(6):1063-70.

39. Hino R, Kabashima K, Kato Y, Yagi H, Nakamura M, Honjo T, et al. Tumor cell expression of programmed cell death-1 ligand 1 is a prognostic factor for malignant melanoma. Cancer. 2010 Apr 1;116(7):1757-66.

40. Kraft S, Fernandez-Figueras M-T, Richarz NA, Flaherty KT, Hoang MP. PDL1 expression in desmoplastic melanoma is associated with tumor aggressiveness and progression. J Am Acad Dermatol. 2017 Sep;77(3):53442.

41. Wang Q, Liu F, Liu L. Prognostic significance of PD-L1 in solid tumor: an updated meta-analysis. Medicine (Baltimore). 2017 May;96(18):e6369.

42. Azimi F, Scolyer RA, Rumcheva P, Moncrieff M, Murali R, McCarthy SW, et al. Tumor-infiltrating lymphocyte grade is an independent predictor of sentinel lymph node status and survival in patients with cutaneous melanoma. J Clin Oncol. 2012 Jul 20;30(21):2678-83.

43. Espinosa E, Márquez-Rodas I, Soria A, Berrocal A, Manzano JL, Gonzalez-Cao $M$, et al. Predictive factors of response to immunotherapy - a review from the Spanish Melanoma Group (GEM). Ann Transl Med. 2017;5(19):389.

44. Ayers M, Lunceford J, Nebozhyn M, Murphy E, Loboda A, Kaufman DR, et al. IFN- $\gamma$-related mRNA profile predicts clinical response to PD-1 blockade. J Clin Invest. 2017 Aug 1;127(8):2930-40.

45. Gibney GT, Weiner LM, Atkins MB. Predictive biomarkers for checkpoint inhibitor-based immunotherapy. Lancet Oncol. 2016 Dec;17(12):e542-51.

46. Carter SL, Cibulskis K, Helman E, McKenna A, Shen H, Zack T, et al. Absolute quantification of somatic DNA alterations in human cancer. Nat Biotechnol. 2012 May;30(5):413-21.

47. Li B, Dewey CN. RSEM: accurate transcript quantification from RNA-Seq data with or without a reference genome. BMC Bioinformatics. 2011 Aug 4;12: 323.

48. Jung M, Kristiansen $G$, Dietrich D. DNA methylation analysis of freecirculating DNA in body fluids. Methods Mol Biol. 2018;1708:621-41.

\section{Publisher's Note}

Springer Nature remains neutral with regard to jurisdictional claims in published maps and institutional affiliations.

Ready to submit your research? Choose BMC and benefit from

- fast, convenient online submission

- thorough peer review by experienced researchers in your field

- rapid publication on acceptance

- support for research data, including large and complex data types

- gold Open Access which fosters wider collaboration and increased citations

- maximum visibility for your research: over $100 \mathrm{M}$ website views per year

At $\mathrm{BMC}$, research is always in progress.

Learn more biomedcentral.com/submission 